\title{
ON THE VOLUMES OF CANONICAL CUSPS OF COMPLEX HYPERBOLIC MANIFOLDS
}

\author{
INKANG KIM AND JOONHYUNG KIM
}

\begin{abstract}
We first introduce a complex hyperbolic space and a complex hyperbolic manifold. After defining the canonical horoball and the canonical cusp on the complex hyperbolic manifold, we estimate the volumes of canonical cusps of complex hyperbolic manifolds. Finally, we deal with cusped, complex hyperbolic 2-manifolds, and in particular, the ones with only one cusp.
\end{abstract}

\section{Introduction}

In [5], J. R. Parker estimates the volumes of canonical cusps of complex hyperbolic manifolds. After dealing with the general case, he improves that result in the case of (complex) dimension 2, and also extends it when the manifold has only one cusp. More concretely, he proves that the volume of the canonical cusp of a complex hyperbolic 2-manifold is at least $2 / 3$, and if that manifold has only one cusp, then at least 1 .

In this paper, we investigate the volumes of canonical cusps of complex hyperbolic 2-manifolds so that we can eliminate one special case, and can make an improvement on the constant from 1 to $4 / 3$.

The rest of this paper is organized as follows: In $\S 2$, we briefly discuss some definitions and properties related to complex hyperbolic spaces. In $\S 3$, we define the canonical horoball and the canonical cusp on a complex hyperbolic manifold. Finally in $\S 4$, we estimate the volumes of canonical cusps of complex hyperbolic manifolds.

\section{Complex hyperbolic space}

\subsection{Siegel domain}

Let $\mathbb{C}^{2,1}$ be a complex vector space of dimension 3 with a Hermitian form of signature $(2,1)$. An element of $\mathbb{C}^{2,1}$ is a column vector $z=\left(z_{1}, z_{2}, z_{3}\right)$. In

Received August 22, 2007; Revised October 23, 2007.

2000 Mathematics Subject Classification. 22E40, 32Q45, 51M10, 57S30.

Key words and phrases. complex hyperbolic manifold, canonical cusp, Heisenberg isometry.

The first author gratefully acknowledges the partial support of KRF grant (KRF-2005015-C00048). 
what follows, we choose the Hermitian form on $\mathbb{C}^{2,1}$ given by the matrix $J$

$$
J=\left[\begin{array}{lll}
0 & 0 & 1 \\
0 & 1 & 0 \\
1 & 0 & 0
\end{array}\right]
$$

Thus $\langle z, w\rangle=w^{*} J z=z_{1} \overline{w_{3}}+z_{2} \overline{w_{2}}+z_{3} \overline{w_{1}}$, where $w^{*}$ is the Hermitian transpose of $w$.

We may choose other matrices like

$$
\left[\begin{array}{ccc}
0 & -1 & 0 \\
-1 & 0 & 0 \\
0 & 0 & 1
\end{array}\right] \text { or }\left[\begin{array}{ccc}
1 & 0 & 0 \\
0 & 1 & 0 \\
0 & 0 & -1
\end{array}\right]
$$

We define the Siegel domain $\mathfrak{S}$ of a complex hyperbolic 2-space $\mathbf{H}_{\mathbb{C}}^{2}$ by identifying points of $\mathfrak{S}$ with their horospherical coordinates, $z=(\zeta, v, u) \in$ $\mathbb{C} \times \mathbb{R} \times \mathbb{R}_{+}$. For each $u>0$, the horosphere of height $u$ is the subset of $\mathfrak{S}$ given by $H_{u}=\mathbb{C} \times \mathbb{R} \times\{u\}$ and the horoball of height $u$ is $B_{u}=\mathbb{C} \times \mathbb{R} \times(u, \infty)$. The boundary of $\mathfrak{S}$ is given by $H_{0} \cup\left\{q_{\infty}\right\}$, where $q_{\infty}$ is a distinguished point at infinity and $H_{0}=\mathbb{C} \times \mathbb{R} \times\{0\}$.

\subsection{Heisenberg group}

The boundary of a complex hyperbolic space is identified with the one point compactification of the Heisenberg group. The 3-dimensional Heisenberg group $\mathfrak{N}$ is $\mathbb{C} \times \mathbb{R}$ with the group law

$$
\left(\zeta_{1}, v_{1}\right) \diamond\left(\zeta_{2}, v_{2}\right)=\left(\zeta_{1}+\zeta_{2}, v_{1}+v_{2}+2 \operatorname{Im}\left(\zeta_{1} \overline{\zeta_{2}}\right)\right)
$$

There is a canonical projection from $\mathfrak{N}$ to $\mathbb{C}$ called the vertical projection given by $\Pi:(\zeta, v) \mapsto \zeta$.

The Heisenberg group acts on itself by Heisenberg translation. For $(\tau, t) \in \mathfrak{N}$, this translation is

$$
T_{(\tau, t)}:(\zeta, v) \mapsto(\zeta+\tau, v+t+2 \operatorname{Im}(\tau \bar{\zeta}))=(\tau, t) \diamond(\zeta, v)
$$

A Heisenberg translation by $(0, t)$ for any $t \in \mathbb{R}$ is called the vertical translation by $t$. One can easily check that the commutator of Heisenberg translations by $(\tau, t)$ and $(\sigma, s)$ is a vertical translation by $4 \operatorname{Im}(\sigma \bar{\tau})$.

In the case of dimension 2, the Heisenberg rotation is just $e^{i \theta}$. (In general, the unitary group $\mathbf{U}(n-1)$ acts on the Heisenberg group by Heisenberg rotation.)

\subsection{Holomorphic isometries}

$$
\begin{gathered}
\text { Define a map } \overline{\mathfrak{S}} \rightarrow \mathbb{P}^{2,1} \text { by } \\
\psi:(\zeta, v, u) \mapsto\left[\begin{array}{c}
\left(-|\zeta|^{2}-u+i v\right) / 2 \\
\zeta \\
1
\end{array}\right] \text { for }(\zeta, v, u) \in \overline{\mathfrak{S}}-\left\{q_{\infty}\right\} ; \psi: q_{\infty} \mapsto\left[\begin{array}{l}
1 \\
0 \\
0
\end{array}\right] .
\end{gathered}
$$


Then $\psi$ maps $\mathfrak{S}$ homeomorphically to the set of points $z$ in $\mathbb{P} \mathbb{C}^{2,1}$ with $\langle z, z\rangle<$ 0 , and maps $\partial \mathfrak{S}$ homeomorphically to the set of points $z$ in $\mathbb{P} \mathbb{C}^{2,1}$ with $\langle z, z\rangle=$ 0 . We write $\psi(\tilde{z})=z$.

The Bergman metric on $\mathfrak{S}$ is given by the distance formula

$$
\cosh ^{2}\left(\frac{\rho(\tilde{z}, \tilde{w})}{2}\right)=\frac{\langle z, w\rangle\langle w, z\rangle}{\langle z, z\rangle\langle w, w\rangle} .
$$

Then the element of volume with the Bergman metric is given by

$$
d \mathrm{Vol}=\frac{4}{u^{3}} d \zeta d v d u
$$

The holomorphic isometry group of $\mathfrak{S}$ with respect to the Bergman metric is the projective unitary group $\mathbf{P} \mathbf{U}(2,1)$ and it acts on $\mathbb{P} \mathbb{C}^{2,1}$ by matrix multiplication.

The action of Heisenberg isometries extends to the Siegel domain, preserving each horosphere and fixing $q_{\infty}$. Some examples of Heisenberg isometries are as follows: The Heisenberg rotation corresponds to the matrix $f \in \mathbf{P} \mathbf{U}(2,1)$, and the Heisenberg translation $T_{(\tau, t)}$ to the matrix $g \in \mathbf{P U}(2,1)$, where

$$
f=\left[\begin{array}{ccc}
1 & 0 & 0 \\
0 & e^{i \theta} & 0 \\
0 & 0 & 1
\end{array}\right] \text { and } g=\left[\begin{array}{ccc}
1 & -\tau^{*} & -|\tau|^{2} / 2+i t / 2 \\
0 & 1 & \tau \\
0 & 0 & 1
\end{array}\right]
$$

When viewed as elements of $\mathbf{P U}(2,1)$, Heisenberg translations are known as pure-parabolic maps and Heisenberg rotations as boundary-elliptic maps. Since discrete subgroups of $\mathbf{P} \mathbf{U}(2,1)$ can only contain elliptic elements of finite order, torsion-free discrete groups cannot contain elliptic elements. A Heisenberg isometry is called screw-parabolic if it is the product of a vertical translation and a Heisenberg rotation. It preserves the fixed-point set of the Heisenberg rotation, called the axis, and acts as a vertical translation there. The screwparabolic map fixing $q_{\infty}$ with axis $(0, v) \subset \mathfrak{N}$, rotation multiplier $e^{i \theta}$, and translation length $t$ is

$$
f=\left[\begin{array}{ccc}
1 & 0 & i t / 2 \\
0 & e^{i \theta} & 0 \\
0 & 0 & 1
\end{array}\right]
$$

\section{Canonical horoballs}

Let $G$ be a discrete subgroup of $\mathbf{P U}(2,1)$ so that the stabiliser $G_{\infty}$ of $q_{\infty}$ contains Heisenberg translations by $(0, t)$ and $\left(\tau, t^{\prime}\right)$, where $t>0$ and $\tau \neq 0$. The horoball $B$ based at $q_{\infty}$ of height

$$
\min \left\{t,\left.|| \tau\right|^{2}+i t / 2 \mid\right\}
$$

is called the canonical horoball at $q_{\infty}$ for $G$.

Let $G$ be a discrete, torsion-free subgroup of $\mathbf{P U}(2,1)$ containing a vertical translation by $t>0$ in the stabiliser $G_{\infty}$ of $q_{\infty}$. The horoball $B$ based at $q_{\infty}$ of 
height $t / 2$ is called the canonical horoball at $q_{\infty}$ for $G$. We define the canonical cusp to be $B / G_{\infty}$.

An important fact on canonical horoballs worth taking note of for our purposes is the following:

Proposition 3.1 (Proposition 5.7 in [2]). Canonical horoballs at distinct parabolic fixed points are disjoint.

\section{Volumes of canonical cusps}

We need two lemmas.

Lemma 4.1 (Lemma 5.2 in [2] or Lemma 1.1 in [5]). Let $G$ be a discrete cocompact group of Heisenberg isometries which acts on $\mathfrak{S}$ fixing $q_{\infty} \in \partial \mathfrak{S}$ as above. Then for any horoball $B_{u}$, we have

$$
\operatorname{Vol}_{\mathfrak{S}}\left(B_{u} / G\right)=\frac{1}{n u^{n}} \operatorname{Vol}_{\mathfrak{N}}(\mathfrak{N} / G)
$$

Lemma 4.2 (Lemma 1.2 in [5]). Let $L$ be a Heisenberg lattice. Suppose that the shortest vertical translation in $L$ is a Heisenberg translation by $(0, t)$, where $t>0$. Then

$$
\operatorname{Vol}_{\mathfrak{N}}(\mathfrak{N} / L)=4 t \operatorname{Vol}_{\mathbb{C}}(\mathbb{C} / \Pi(L)) .
$$

\subsection{Cusped, complex hyperbolic $n$-manifolds}

As we can see from the following theorem, J. R. Parker in [5], estimates the volumes of canonical cusps of complex hyperbolic $n$-manifolds.

Theorem 4.3 (Theorem 3.1 in [5]). Let $G$ be a discrete, torsion-free, cofinitevolume group of isometries of $\mathbf{H}_{\mathbb{C}}^{n}, q_{\infty}$ a parabolic fixed point of $G$, and $G_{\infty}$ its stabiliser in $G$. Suppose that $G_{\infty}$ contains a Heisenberg lattice $L$ as a subgroup of index $m$. Let $B$ be the canonical horoball based at $q_{\infty}$. Then $\operatorname{Vol}_{\mathfrak{S}}\left(B / G_{\infty}\right) \geq 2^{n} / n m$.

Corollary 4.4 (Corollary 3.2 in [5]). Let $M$ be a complex hyperbolic $n$-manifold of finite volume with $k$ ends. Let $I_{n}$, which is at most $2(6 \pi)^{(2 n-1)(n-1)}$, be the largest index of a lattice in the fundamental group of a compact Heisenberg $(2 n-1)$-manifold. Then $\operatorname{Vol}_{\mathfrak{S}}(M) \geq 2^{n} k / n I_{n}$.

In [3], by using techniques of algebraic geometry, J.-M. Hwang improves the above volume constants. But his result tells nothing about canonical cusps.

Theorem 4.5 (Theorem 1.2 in [3]). Following the notations of the result above, for $n \geq 2$,

$\operatorname{Vol}_{\mathfrak{S}}(M) \geq \frac{(4 \pi)^{n} k}{n !(P(4)-P(2))}\left(1-\frac{n+1}{P(4)-P(2)}\right)$, where $P(\ell):=\frac{(n \ell+n+\ell) !}{n !(n \ell+\ell) !}$. 


\subsection{Cusped, complex hyperbolic 2-manifolds}

In the case of $n=2$, Theorem 4.3 says that $\operatorname{Vol}_{\mathfrak{S}}\left(B / G_{\infty}\right) \geq 2 / m$. Moreover, S. Hersonsky and F. Paulin proves that $I_{2}=6$ (Proposition 5.8 in [2]), so $m \in\{2,3,4,6\}$ because after a vertical projection, the order of the symmetry of the Heisenberg lattice is the same as the plane lattice. That is, the number $m$ is just the order of the rotational symmetry of a lattice in $\mathbb{C}$. It is the so-called crystallographic restriction. Hence, Theorem 4.3 says that $\operatorname{Vol}_{\mathfrak{S}}\left(B / G_{\infty}\right) \geq 1 / 3$ when $n=2$. But J. R. Parker improves this constant from $1 / 3$ to $2 / 3$.

Theorem 4.6 (Theorem 5.1 in [5]). Let $G$ be a discrete, torsion-free, cofinitevolume group of isometries of $\mathbf{H}_{\mathbb{C}}^{2}, q_{\infty}$ a parabolic fixed point of $G$, and $G_{\infty}$ its stabiliser in $G$. Let $B$ be the canonical horoball based at $q_{\infty}$. Then

$$
\operatorname{Vol}_{\mathfrak{S}}\left(B / G_{\infty}\right) \geq 2 / 3 .
$$

Corollary 4.7 (Corollary 5.2 in [5]). Let $M$ be a complex hyperbolic 2-manifold with $k$ ends. Then $\operatorname{Vol}_{\mathfrak{S}}(M) \geq 2 k / 3$.

Remark 4.8. J.-M. Hwang's result (Theorem 4.5) is better than J. R. Parker's one, too. When we set $n=2$ in Theorem 4.5 , we get $\operatorname{Vol}_{\mathfrak{S}}(M) \geq \frac{160}{1323} \pi^{2} k \geq$ $1.19 \cdot k$.

We will give a sketch of the proof of the above theorem ([5]). Let $g$ be the shortest vertical translation by $t>0$ in $G_{\infty}$, and $L$ a Heisenberg lattice. Then $\Pi(L)$ becomes a lattice in $\mathbb{C}$. Suppose that $\tau$ has the shortest length among all nontrivial elements of $\Pi(L)$ and that $\sigma$ has the shortest length among all elements of $\Pi(L)$ that are not real multiples of $\tau$. Then $\{\tau, \sigma\}$ becomes a basis for $\Pi(L)$ and $\sigma / \tau$ is in the standard fundamental region for the modular group. If we pullback the translations by $\tau$ and $\sigma$ under $\Pi$, they become Heisenberg translation in $L$ by $\left(\tau, t^{\prime}\right)$ and $\left(\sigma, s^{\prime}\right)$ for some $t^{\prime}, s^{\prime} \in \mathbb{R}$. Then, as mentioned in $\S 2.2$, their commutator is a vertical translation by $4 \operatorname{Im}(\sigma \bar{\tau})=t p$ for some $p \in \mathbb{N}$.

Now we can calculate the volumes of canonical cusps of complex hyperbolic manifolds. The area of a fundamental domain for $\Pi(L)$ is $\operatorname{Im}(\sigma \bar{\tau})=t p / 4$, so by Lemma 4.2 , we have

$$
\operatorname{Vol}_{\mathfrak{N}}(\mathfrak{N} / L)=4 t \operatorname{Vol}_{\mathbb{C}}(\mathbb{C} / \Pi(L))=4 t(t p / 4)=t^{2} p .
$$

If we let $L$ have index $m$ in $G_{\infty}$,

$$
\operatorname{Vol}_{\mathfrak{N}}\left(\mathfrak{N} / G_{\infty}\right)=t^{2} p / m \text {. }
$$

Moreover, since the canonical horoball $B$ has height

$$
\min \left\{t,\left.|| \tau\right|^{2}+i t / 2 \mid\right\},
$$

by Lemma 4.1 ,

$$
\operatorname{Vol}_{\mathfrak{S}}\left(B / G_{\infty}\right)=\frac{1}{2 u^{2}} \operatorname{Vol}_{\mathfrak{N}}\left(\mathfrak{N} / G_{\infty}\right)=\frac{1}{2 u^{2}} \frac{t^{2} p}{m}
$$


As $G$ is torsion-free, the canonical horoball has height $u=\frac{t}{2}$, and so,

$$
\operatorname{Vol}_{\mathfrak{S}}\left(B / G_{\infty}\right)=\frac{1}{2 u^{2}} \frac{t^{2} p}{m}=\frac{2 p}{m} .
$$

If $m=2$ or 3 , then $2 p / m \geq 2 / 3$. Since J. R. Parker proves the following theorem, which says that if $m=4$ or 6 , then $p \neq 1,2 p / m \geq 2 / 3$. This proves the above theorem.

Theorem 4.9 (Proposition 5.5 in [5]). Let $G$ be a discrete, torsion-free, cocompact group of Heisenberg isometries. Suppose that $G$ contains a screw-parabolic map $f$ whose rotational part has order $m=4$ or 6 . Let $k$ be the shortest vertical translation in $G$, and let $g$ be a shortest translation in $\Pi(G)$. Now $\left[f g f^{-1}, g\right]$ is a vertical translation, and so $\left[f g f^{-1}, g\right]=k^{ \pm p}$, where $p$ is a positive integer. Then $p \geq 2$.

J. R. Parker proves this theorem using the following two lemmas.

Lemma 4.10 (Lemma 5.3 in [5]). Let $G$ be a discrete, torsion-free group of Heisenberg isometries containing the screw-parabolic map $f$ with rotational part of order $m=2,3,4$, or 6 . Let $k$, the vertical translation by $t>0$, be the shortest vertical translation in $G$. Then there is an integer a so that the screw-parabolic map $f k^{-a}$ is also in $G$, has the same rotation multiplier as $f$, and has the property that $\left(f k^{-a}\right)^{m}=k^{ \pm 1}$. In other words, the translation length of $f k^{-a}$ is $\pm t / m$.

Lemma 4.11 (Lemma 5.4 in [5]). Let $f$ be a screw-parabolic Heisenberg isometry with axis $(o, v) \subset \mathfrak{N}$, rotation multiplier $e^{i \theta}$, and translation length $a \in \mathbb{R}$. Let $g$ be the Heisenberg translation by $(\sigma, s) \in \mathfrak{N}$. Then $g f$ is a screw-parabolic map with axis $\left(\sigma /\left(1-e^{i \theta}\right), v\right)$, rotational multiplier $e^{i \theta}$, and translation length $b=a+s-|\sigma|^{2} \sin \theta /(1-\cos \theta)$.

Now we are ready to state the main result of the paper. It says that if $m=2$, then $p$ must be even in the above situation.

Theorem 4.12 (Main result). Let $G$ be a discrete, torsion-free, cocompact group of Heisenberg isometries. Suppose that $G$ contains a screw-parabolic map $f$ whose rotational part has order $m=2$. Then $p$ must be an even integer, where $p$ is defined as above.

Proof. By Lemma 4.10, we may assume that $G$ contains $g$, a Heisenberg translation by $(\sigma, s)$ and $f$, a screw-parabolic with axis $(o, v) \subset \mathfrak{N}$, rotation multiplier -1 , and translation length $a= \pm t / 2$. We may also assume that $G$ contains $h$, a Heisenberg translation by $\left(\tau, s^{\prime}\right)$ so that

$$
[g, h]=\left[\begin{array}{ccc}
1 & 0 & -\sigma^{*} \tau+\tau^{*} \sigma \\
0 & 1 & 0 \\
0 & 0 & 1
\end{array}\right],
$$

which is a vertical translation by $4 \operatorname{Im}(\sigma \bar{\tau})=2 i(\bar{\sigma} \tau-\sigma \bar{\tau})=p t$. 
Since $m=2, \Pi\left(G_{\infty}\right)$ is a $(2,2,2,2)$-group and order 2 elliptic conjugacy classes are projections of $f, g f, h f$, and $h g f$. (See Section 5.5, Table 2 in [6] and Section V. D. 9 in [4].) By Lemma 4.11, the translation length of $g f$ is $b=a+s= \pm t / 2+s$ and that of $h f$ is $c=a+s^{\prime}= \pm t / 2+s^{\prime}$. Since $(g f)^{2}$ is a vertical translation, $2 b= \pm t+2 s$ is an integer multiple of $t$, so $s=l t / 2$ for some integer $l$. But if $l$ is an odd integer, the translation length of $g f$ is an integer of $t$, i.e., $b=r t$ for some integer $r$. If we let $k$ be the shortest vertical translation in $G$, then $(g f) k^{-r}$ is a Heisenberg translation, so $G$ cannot be torsion free. Thus $s=l^{\prime} t$. By applying a similar argument to $h f$, we get that $s^{\prime}=l^{\prime \prime} t$. Furthermore, the translation length of $h g f$ is $d=a+s+s^{\prime}-p t / 2= \pm t / 2+l^{\prime} t+l^{\prime \prime} t-p t / 2$ since $h g$ is a Heisenberg translation by $\left(\tau+\sigma, s^{\prime}+s-p t / 2\right)$. If $p$ is an odd integer, then $d=x t$ for some integer $x$. This means that $(h g f) k^{-x}$ is a Heisenberg rotation, so $G$ cannot be torsion free. Therefore $p$ must be an even integer.

As mentioned above, if $m=4$ or 6 , then $p \neq 1$. The following theorem is a generalization.

Theorem 4.13. Let $G$ be a discrete, torsion-free, cocompact group of Heisenberg isometries. Suppose that $G$ contains a screw-parabolic map $f$ whose rotational part has order $m=4$ or 6 . Let $k$ be the shortest vertical translation in $G$, and let $g$ be a shortest translation in $\Pi(G)$. Now $\left[f g f^{-1}, g\right]$ is a vertical translation, and so $\left[f g f^{-1}, g\right]=k^{ \pm p}$, where $p$ is a positive integer. Then $p$ must be an even integer.

Proof. The proof is similar to that of Proposition 5.5 in [5]. Suppose that $g$ is a Heisenberg translation by $(\sigma, s)$, the axis of $f$ is $(o, v) \subset \mathfrak{N}$, the multiplier of $f$ is $e^{2 i \pi / m}$, and the translation length of $f$ is $a$. Then $f g f^{-1}$ is a Heisenberg translation by $\left(e^{2 i \pi / m} \sigma, s\right)$, so $\left[f g f^{-1}, g\right]$ is a vertical translation by $4|\sigma|^{2} \sin (2 \pi / m)=p t$ (where $t$ is the translation length of $k$ ). By Lemma 4.10, we may assume that $a= \pm t / m= \pm 4|\sigma|^{2} \sin (2 \pi / m) / p m$.

Suppose that $m=4$, i.e., $4|\sigma|^{2}=p t$. Then $\Pi\left(G_{\infty}\right)$ is a $(2,4,4)$-triangle group and the generators are $f, g f$, and $g f^{2}$, where the orders are 4,4 , and 2 , respectively. (See Section 5.5, Table 2 in [6] and Section V. D. 9 in [4].) We already know that $f$ has a rotational multiplier $e^{2 \pi i / 4}=i$, and a translation length $a= \pm t / 4$. Using Lemma 4.11, we get the rotational multipliers and translation lengths of screw-parabolic maps $g f$ and $g f^{2}$. The generator $g f$ has a rotational multiplier $i$, and a translation length $a+s-|\sigma|^{2}= \pm t / 4+s-p t / 4$. On the other hand, $g f^{2}$ has a rotational multiplier $e^{\pi i}=-1$, and a translation length $2 a+s= \pm t / 2+s$. Since $g f^{2}$ is order $2,2( \pm t / 2+s)= \pm t+2 s$ is an integer multiple of $t$, so $s=l^{\prime} t / 2$ for some integer $l^{\prime}$. But since $G$ is torsionfree, $\pm t / 2+s$ cannot be an integer multiple of $t$ by the same argument used in the proof of Theorem 4.12. Hence, $l^{\prime}$ must be an even integer, i.e., $s=l t$ for some integer $l$. If $p$ is an odd integer, then the translation length of $(g f)^{2}$ is 
$2( \pm t / 4+s-p t / 4)= \pm t / 2+2 l t-p t / 2$ which is an integer multiple of $t$, and that contradicts the fact that $G$ is torsion-free. So $p$ must be an even integer.

Now suppose that $m=6$, i.e., $2|\sigma| \sqrt{3}=p t$. This proof is almost the same as when $m=4$. Then $\Pi\left(G_{\infty}\right)$ is a $(2,3,6)$-triangle group and the generators are $f, g f^{2}$, and $g f^{3}$, where the orders are 6, 3, and 2, respectively. (See also Section 5.5, Table 2 in [6] and Section V. D. 9 in [4] as above.) We already know that $f$ has a rotational multiplier $e^{2 \pi i / 6}=e^{\pi i / 3}$, and a translation length $a= \pm t / 6$. Using Lemma 4.11, we get the rotational multipliers and translation lengths of screw-parabolic maps $g f^{2}$ and $g f^{3}$. The generator $g f^{2}$ has a rotational multiplier $e^{2 \pi i / 3}$, and a translation length $2 a+s-|\sigma|^{2} / \sqrt{3}=$ $\pm t / 3+s-p t / 6$. On the other hand, $g f^{3}$ has a rotational multiplier $e^{\pi i}=-1$, and a translation length $3 a+s= \pm t / 2+s$. Since $g f^{3}$ is order $2,2( \pm t / 2+s)=$ $\pm t+2 s$ is an integer multiple of $t$, so $s=l^{\prime} t / 2$ for some integer $l^{\prime}$. But since $G$ is torsion-free, $\pm t / 2+s$ cannot be an integer multiple of $t$. Hence $s=l t$ for some integer $l$, and since $g f^{2}$ is of order 3, the translation length of $\left(g f^{2}\right)^{3}$ is $3( \pm t / 3+s-p t / 6)= \pm t+3 l t-p t / 2$ must be an integer multiple of $t$. Hence $p$ must be an even integer.

\subsection{One-ended, cusped, complex hyperbolic 2-manifolds}

In this section, we deal with one-ended, cusped, complex hyperbolic 2manifolds. In this special case, J. R. Parker improves the above constant $2 / 3$ in Theorem 4.6 to 1 in [5].

Theorem 4.14 (Theorem 6.1 in [5]). Let $G$ be a discrete, torsion-free, cofinitevolume group of isometries of $\mathbf{H}_{\mathbb{C}}^{2}, q_{\infty}$ a parabolic fixed point of $G$, and $G_{\infty}$ its stabiliser in $G$. Suppose that $G_{\infty}$ contains representatives for all the parabolic conjugacy classes in $G$. Then there is a precisely invariant horoball $B^{\prime}$ based at $q_{\infty}$ so that $\operatorname{Vol}_{\mathfrak{S}}\left(B^{\prime} / G_{\infty}\right) \geq 1$.

The method of proof is as follows: Except for the cases $m=3, p=1$ and $m=6, p=2, \operatorname{Vol}_{\mathfrak{S}}\left(B^{\prime} / G_{\infty}\right)=2 p / m \geq 1$. So he considers these two cases separately and finds larger horoballs that are precisely invariant under $G_{\infty}$ in $G$.

If $m=3$ and $p=1$, the horoball of height $t / 3$ is precisely invariant un$\operatorname{der} G_{\infty}$ in $G$, so $\operatorname{Vol}_{\mathfrak{S}}\left(B^{\prime} / G_{\infty}\right)=3 / 2$. For the second case, $m=6$ and $p=2$, the horoball of height $t / 2 \sqrt{3}$ is precisely invariant under $G_{\infty}$ in $G$, so $\operatorname{Vol}_{\mathfrak{S}}\left(B^{\prime} / G_{\infty}\right)=2$.

Now, we get the following result as a corollary of our main result. This corollary improves the constant 1 in the above theorem to $4 / 3$.

Corollary 4.15 (Corollary of Theorem 4.12). Let $G$ be a discrete, torsion-free, cofinite-volume group of isometries of $\mathbf{H}_{\mathbb{C}}^{2}, q_{\infty}$ a parabolic fixed point of $G$, and $G_{\infty}$ its stabiliser in $G$. Suppose that $G_{\infty}$ contains representatives for all the parabolic conjugacy classes in $G$. Then there is a precisely invariant horoball $B^{\prime}$ based at $q_{\infty}$ so that $\operatorname{Vol}_{\mathfrak{S}}\left(B^{\prime} / G_{\infty}\right) \geq 4 / 3$. 
Remark 4.16. When considering the volumes of complex hyperbolic manifolds with only one cusp, not just a canonical cusp, J.-M. Hwang's result in Theorem 4.5 is still better than that of J. R. Parker in Theorem 4.14. But our result $4 / 3$ is better than J.-M. Hwang's result, at least in the one-cusped case.

Remark 4.17. In Theorem 4.14, equality holds only if $m=2$ and $p=1$, but Theorem 4.12 excludes that case. Now to improve the estimate of Corollary 4.15 above, one needs only consider the following three cases: $(m, p)=(3,2),(4,2)$, and $(6,4)$.

Proof. 1) If $m=6$, then by Theorem 4.13, $p \neq 1,3$. Now by Theorem 4.14, when $p=2, \operatorname{Vol}_{\mathfrak{S}}\left(B^{\prime} / G_{\infty}\right)=2 \geq 4 / 3$. Clearly when $p \geq 4, \operatorname{Vol}_{\mathfrak{S}}\left(B^{\prime} / G_{\infty}\right) \geq$ $4 / 3$.

2) If $m=4$, then by Theorem 4.13, $p \neq 1$. Now when $p=2$, we can prove that $\operatorname{Vol}_{\mathfrak{S}}\left(B^{\prime} / G_{\infty}\right)=4 / 3$ the way we proved Theorem 4.14. (See the last remark in [5].) Clearly when $p \geq 3, \operatorname{Vol}_{\mathfrak{S}}\left(B^{\prime} / G_{\infty}\right) \geq 4 / 3$.

3 ) If $m=3$ and $p=1$, then by Theorem $4.14, \operatorname{Vol}_{\mathfrak{S}}\left(B^{\prime} / G_{\infty}\right)=3 / 2 \geq 4 / 3$. Now clearly, when $p \geq 2, \operatorname{Vol}_{\mathfrak{S}}\left(B^{\prime} / G_{\infty}\right) \geq 4 / 3$.

4) If $m=2$, then by Theorem $4.12, p \neq 1$. Now when $p \geq 2, \operatorname{Vol}_{\mathfrak{S}}\left(B^{\prime} / G_{\infty}\right) \geq$ 2. This completes the whole proof.

Acknowledgement. The authors wish to thank an anonymous referee for pointing out some inaccuracies in the first draft of the manuscript.

\section{References}

[1] W. M. Goldman, Complex Hyperbolic Geometry, Oxford Mathematical Monographs. Oxford Science Publications. The Clarendon Press, Oxford University Press, New York, 1999.

[2] S. Hersonsky and F. Paulin, On the volumes of complex hyperbolic manifolds, Duke Math. J. 84 (1996), no. 3, 719-737.

[3] J.-M. Hwang, On the volumes of complex hyperbolic manifolds with cusps, Internat. J. Math. 15 (2004), no. 6, 567-572.

[4] B. Maskit, Kleinian Groups, Grundlehren der Mathematischen Wissenschaften [Fundamental Principles of Mathematical Sciences], 287. Springer-Verlag, Berlin, 1988.

[5] J. R. Parker, On the volumes of cusped, complex hyperbolic manifolds and orbifolds, Duke Math. J. 94 (1998), no. 3, 433-464.

[6] W. Thurston, The geometry and topology of 3-manifolds, preprint, 1991.

INKANG KIM

DEPARTMENT OF MATHEMATICS

Seoul National University

SEOUL 151-747, KOREA

E-mail address: inkang@snu.ac.kr

JOONHYUNG KIM

DEPARTMENT OF MATHEMATICS

Seoul National University

SEOUL 151-747, KorEA

E-mail address: calvary@snu.ac.kr 